\title{
Sliding Mode Control of the Fractional-Order Unified Chaotic System
}

\author{
Jian Yuan, Bao Shi, Xiaoyun Zeng, Wenqiang Ji, and Tetie Pan \\ Institute of Systems Science and Mathematics, Naval Aeronautical and Astronautical University, Yantai, Shandong 264001, China \\ Correspondence should be addressed to Jian Yuan; yuanjianscar@gmail.com
}

Received 26 June 2013; Revised 9 September 2013; Accepted 9 September 2013

Academic Editor: Dumitru Baleanu

Copyright (C) 2013 Jian Yuan et al. This is an open access article distributed under the Creative Commons Attribution License, which permits unrestricted use, distribution, and reproduction in any medium, provided the original work is properly cited.

\begin{abstract}
This paper deals with robust synchronization of the fractional-order unified chaotic systems. Firstly, control design for synchronization of nominal systems is proposed via fractional sliding mode technique. Then, systematic uncertainties and external disturbances are considered in the fractional-order unified chaotic systems, and adaptive sliding mode control is designed for the synchronization issue. Finally, numerical simulations are carried out to verify the effectiveness of the two proposed control techniques.
\end{abstract}

\section{Introduction}

Even though the theory of fractional calculus dates back to the end of the 17th century, the subject only really came to life over the last few decades [1]. The most significant advantage of fractional calculus is that it provides a powerful instrument of describing memory and hereditary properties of different substances [2]. In particular, fractional differential equations, as the basic theory for fractional-order control [3], have become a powerful tool in describing the dynamics of complex systems and gained great development very recently [4-6].

One of the most important areas of application is the fractional-order chaotic systems, which have wide potential applications in engineering. Since Hartley et al. firstly discovered chaotic phenomenon in fractional dynamics systems [7], there has emerged great interest in this novel and promising topic. On one hand, more and more fractional nonlinear systems which exhibit chaos have been discovered, and their chaotic behaviors have been studied with numerical simulations, such as the fractional-order Chua circuit [8], the fractional-order Van der Pol oscillator [9-11], the fractional-order Lorenz system [12, 13], the fractionalorder Chen system [14-16], the fractional-order Lü system [17], the fractional-order Liu system [18], the fractional-order
Rössler system [19, 20], the fractional-order Arneodo system [21], the fractional-order Lotka-Volterra system [22, 23], the fractional-order financial system $[24,25]$, and the discrete fractional logistic map [26]. On the other hand, control and synchronization of fractional-order dynamical systems have been attracting growing investigations. Linear-state feedback control approach has been designed in [14, 27-35], nonlinear feedback control in [36-40], fractional PID control in [41, 42], and open-plus-closed-loop control in [43]. To tackle with modeling inaccuracies and external noises which are unavoidable in the real-world application, fractional-order sliding mode control methodology has been established in [44-51].

In this paper, we investigate robust synchronization of the fractional-order unified chaotic systems. We firstly propose controllers to synchronize the nominal systems via fractional sliding mode technique. Secondly, we consider systematic uncertainties and external disturbances in the fractionalorder unified chaotic systems and establish adaptive sliding mode control for synchronization of the uncertain systems.

The rest of this paper is organized as follows. Section 2 presents some basic definitions and theorems about fractional calculus and fractional-order dynamical system. Section 3 describes the general form of fractional-order unified chaotic system and presents our main objective in this 
paper. Section 4 proposes the sliding mode control design for synchronization of nominal systems and adaptive sliding mode control design for the uncertain system. Numerical simulations are presented to show the effectiveness of the proposed schemes in Section 5. Finally, this paper is concluded in Section 6.

\section{Preliminaries}

Definition 1. The most important function used in fractional calculus is Euler's Gamma function, which is defined as

$$
\Gamma(n)=\int_{0}^{\infty} t^{n-1} e^{-t} d t
$$

Definition 2. Another important function is a two-parameter function of the Mittag-Leffler type defined as

$$
E_{\alpha, \beta}(z)=\sum_{k=0}^{\infty} \frac{z^{k}}{\Gamma(\alpha k+\beta)}, \quad \alpha>0, \beta>0 .
$$

Fractional calculus is a generalization of integration and differentiation to noninteger-order fundamental operator ${ }_{a} D_{t}^{\alpha}$, where $a$ and $t$ are the bounds of the operation and $a \in \mathbb{R}$. The continuous integrodifferential operator is defined as

$$
{ }_{a} D_{t}^{\alpha}= \begin{cases}\frac{d^{\alpha}}{d t^{\alpha}}, & \alpha>0 \\ 1, & \alpha=0 \\ \int_{a}^{t}(d \tau)^{\alpha}, & \alpha<0\end{cases}
$$

The three most frequently used definitions for the general fractional calculus are the Grünwald-Letnikov definition, the Riemann-Liouville definition, and the Caputo definition [2, $23,52,53]$.

Definition 3. The Grünwald-Letnikov derivative definition of order $\alpha$ is described as

$$
{ }_{a} D_{t}^{\alpha} f(t)=\lim _{h \rightarrow 0} \frac{1}{h^{\alpha}} \sum_{j=0}^{\infty}(-1)^{j}\left(\begin{array}{c}
\alpha \\
j
\end{array}\right) f(t-j h) .
$$

For binomial coefficients calculation, we can use the relation between Euler's Gamma function and factorial defined as

$$
\left(\begin{array}{c}
\alpha \\
j
\end{array}\right)=\frac{\alpha !}{j !(\alpha-j) !}=\frac{\Gamma(\alpha+1)}{\Gamma(j+1) \Gamma(\alpha-j+1)}
$$

for

$$
\left(\begin{array}{l}
\alpha \\
0
\end{array}\right)=1
$$

Definition 4. The Riemann-Liouville derivative definition of order $\alpha$ is described as

$$
{ }_{a} D_{t}^{\alpha} f(t)=\frac{1}{\Gamma(n-\alpha)} \frac{d^{n}}{d t^{n}} \int_{a}^{t} \frac{f(\tau) d \tau}{(t-\tau)^{\alpha-n+1}}, \quad n-1<\alpha<n .
$$

However, applied problems require definitions of fractional derivatives allowing the utilization of physically interpretable initial conditions, which contain $f(a), f^{\prime}(a)$, and so forth. Unfortunately, the Riemann-Liouville approach fails to meet this practical need. It is M. Caputo who solved this conflict.

Definition 5. The Caputo definition of fractional derivative can be written as

$$
{ }_{a} D_{t}^{\alpha} f(t)=\frac{1}{\Gamma(n-\alpha)} \int_{a}^{t} \frac{f^{(n)}(\tau) d \tau}{(t-\tau)^{\alpha-n+1}}, \quad n-1<\alpha<n .
$$

In the following, we use the Caputo approach to describe the fractional chaotic systems and the Grünwald-Letnikov approach to propose numerical simulations. To simplify the notation, we denote the fractional-order derivative as $D^{\alpha}$ instead of ${ }_{0} D_{t}^{\alpha}$ in this paper.

Lemma 6 (see [22]). Consider the following commensurate fractional-order dynamics system:

$$
D^{\alpha} x=f(x),
$$

where $0<\alpha \leq 1$ and $x \in \mathbb{R}^{n}$. The equilibrium points of system (9) are calculated by solving the following equation:

$$
f(x)=0 .
$$

These points are locally asymptotically stable if all eigenvalues $\lambda_{i}$ of the Jacobian matrix $J=\partial f / \partial x$ evaluated at the equilibrium points satisfy

$$
|\arg (\lambda)|>\frac{\alpha \pi}{2}
$$

Lemma 7 (see [22]). Consider the following $n$-dimensional linear fractional-order dynamics system:

$$
\begin{gathered}
D^{q_{1}} x_{1}=a_{11} x_{1}+a_{12} x_{2}+\cdots+a_{1 n} x_{n}, \\
D^{q_{2}} x_{2}=a_{21} x_{1}+a_{22} x_{2}+\cdots+a_{2 n} x_{n}, \\
\cdots \\
D^{q_{n}} x_{n}=a_{n 1} x_{1}+a_{n 2} x_{2}+\cdots+a_{n n} x_{n},
\end{gathered}
$$

where all $\alpha_{i}$ 's are rational numbers between 0 and 1 . Assume $M$ be the lowest common multiple of the denominators $u_{i}$ 's of $\alpha_{i}$ 's, where $\alpha_{i}=v_{i} / u_{i},\left(u_{i}, v_{i}\right)=1$, and $u_{i}, v_{i} \in Z^{+}$, for $i=$ $1,2, \ldots, n$. Define

$$
\Delta(\lambda)=\left(\begin{array}{cccc}
\lambda^{M \alpha_{1}}-a_{11} & -a_{12} & \cdots & -a_{1 n} \\
-a_{21} & \lambda^{M \alpha_{2}}-a_{22} & \cdots & -a_{2 n} \\
\vdots & \vdots & \ddots & \vdots \\
-a_{n 1} & -a_{n 2} & \cdots & \lambda^{M \alpha_{n}}-a_{n n}
\end{array}\right) .
$$

Then, the zero solution of system (12) is globally asymptotically stable in the Lyapunov sense if all roots of the equation $\operatorname{det}(\Delta(\lambda))=0$ satisfy $|\arg (\lambda)|>\pi / 2 M$. 
Lemma 8 (see [53]). Assume that there exists a scalar function $V$ of the state $x$ with continuous first-order derivative such that the following are given:

(i) $V(x)$ is positive definite,

(ii) $\dot{V}(x)$ is negative definite,

(iii) $V(x) \rightarrow \infty$ as $\|x\| \rightarrow \infty$.

Then, the equilibrium at the origin is globally asymptotically stable.

\section{Problem Formulation}

In [54], Lü et al. have considered a kind of chaotic systems and pointed out that these systems can be described in a unified form as follows:

$$
\begin{gathered}
\dot{x}_{1}=(25 \alpha+10)\left(x_{2}-x_{1}\right), \\
\dot{x}_{2}=(28-35 \alpha) x_{1}-x_{1} x_{3}+(29 \alpha-1) x_{2}, \\
\dot{x}_{3}=x_{1} x_{2}-\frac{(8+\alpha) x_{3}}{3},
\end{gathered}
$$

where $x_{1}, x_{2}$, and $x_{3}$ are state variables and $\alpha \in[0,1]$ is the system parameter. Lü et al. [54] call system (14) a unified chaotic system because it is chaotic for any $\alpha \in[0,1]$. When $\alpha \in[0,0.8)$, system (14) is called a the generalized Lorenz chaotic system. When $\alpha=0.8$, it is called the Lü chaotic system. And it is called the generalized Chen chaotic system when $\alpha \in(0.8,1]$.

The fractional-order unified chaotic system has been firstly introduced and studied in [55] and reads as

$$
\begin{gathered}
D^{q_{1}} x_{1}=(25 \alpha+10)\left(x_{2}-x_{1}\right), \\
D^{q_{2}} x_{2}=(28-35 \alpha) x_{1}-x_{1} x_{3}+(29 \alpha-1) x_{2}, \\
D^{q_{3}} x_{3}=x_{1} x_{2}-\frac{(8+\alpha) x_{3}}{3},
\end{gathered}
$$

where $q_{1}, q_{2}, q_{3} \in(0,1]$ is the fractional order.

System (15) is considered as the drive (master) system and the response (slave) system is a controlled system as follows:

$$
\begin{gathered}
D^{q_{1}} y_{1}=(25 \alpha+10)\left(y_{2}-y_{1}\right)+u_{1}, \\
D^{q_{2}} y_{2}=(28-35 \alpha) y_{1}-y_{1} y_{3}+(29 \alpha-1) y_{2}+u_{2}, \\
D^{q_{3}} y_{3}=y_{1} y_{2}-\frac{(8+\alpha) y_{3}}{3}+u_{3} .
\end{gathered}
$$

Let us define the state errors between the response system (16) and the drive system (15) as $e_{1}=y_{1}-x_{1}, e_{2}=y_{2}-x_{2}$, and $e_{3}=y_{3}-x_{3}$.

By subtracting (15) from (16), one can get the following error dynamical system:

$$
\begin{aligned}
& D^{q_{1}} e_{1}=(25 \alpha+10)\left(e_{2}-e_{1}\right)+u_{1}, \\
& D^{q_{2}} e_{2}=(28-35 \alpha) e_{1}+e_{1} e_{3}-e_{1} y_{3} \\
& -e_{3} y_{1}+(29 \alpha-1) e_{2}+u_{2} \\
& D^{q_{3}} e_{3}=-e_{1} e_{2}+e_{1} y_{2}+e_{2} y_{1}-\frac{(8+\alpha) e_{3}}{3}+u_{3} \text {. }
\end{aligned}
$$

Our main objective in this paper is to investigate the synchronization issue for the fractional-order unified chaotic system (15). It is clear that the synchronization of systems (15) and (16) is equivalent to the stabilization of the error dynamical system (17).

\section{Synchronization Design}

In the following, the sliding mode control technique, which can maintain low sensitivity to unmodeled dynamics and external disturbances, is applied to establish an effective control law to guarantee the synchronization of the drive system (15) and the response system (16). Two major steps are involved in the sliding mode control design: firstly, constructing an appropriate sliding surface on which the desired system dynamics is stable and, secondly, developing a suitable control law such that the sliding condition is attained.

4.1. Synchronization of the Nominal System. In this subsection, let us firstly consider a simple case: the nominal fractional-order unified chaotic system; that is, the system contains no systematic uncertainties or external disturbances. The design procedure is elaborated in the rest part of this subsection.

4.1.1. Sliding Surfaces Design. In order to achieve the stability of system (17), three sliding surfaces $S_{1}, S_{2}$, and $S_{3}$ are introduced as

$$
s_{i}(t)=\left(D^{q_{i}}+\lambda_{i}\right) \int_{0}^{t} e_{i}(\tau) d \tau, \quad i=1,2,3,
$$

the time derivative of which becomes

$$
\dot{s}_{i}(t)=D^{q_{i}} e_{i}(t)+\lambda_{i} e_{i}(t), \quad i=1,2,3 .
$$

As long as system (17) operates on the sliding surfaces, it satisfies $s_{i}=0$ and $\dot{s}_{i}=0, i=1,2,3$, which yields the following sliding mode dynamics:

$$
\begin{aligned}
& D^{q_{1}} e_{1}=-\lambda_{1} e_{1}, \\
& D^{q_{2}} e_{2}=-\lambda_{2} e_{2}, \\
& D^{q_{3}} e_{3}=-\lambda_{3} e_{3} .
\end{aligned}
$$

By using Lemmas 6 or 7, system (20) is asymptotically stable. As a result, the sliding mode surfaces (18) we have just constructed are appropriate for the control design.

\subsubsection{Control Laws Design}

Step 1. Choose the first control Lyapunov function

$$
V_{1}(t)=\frac{1}{2} s_{1}^{2}
$$

Taking time derivative gives

$$
\dot{V}_{1}(t)=s_{1} \dot{s}_{1}=s_{1}\left(D^{q_{1}} e_{1}+\lambda_{1} e_{1}\right) .
$$


Substituting the first state equation of (17) into (22), one has

$$
\dot{V}_{1}(t)=s_{1}\left[(25 \alpha+10)\left(e_{2}-e_{1}\right)+u_{1}+\lambda_{1} e_{1}\right] \text {. }
$$

Therefore, by designing the first control law as

$$
u_{1}=-(25 \alpha+10)\left(e_{2}-e_{1}\right)-\lambda_{1} e_{1}-k_{1} \operatorname{sgn}\left(s_{1}\right),
$$

where $k_{1}>0$ and

$$
\operatorname{sgn}(s)= \begin{cases}1, & s>0 \\ 0, & s=0 \\ -1, & s<0\end{cases}
$$

then, (23) becomes

$$
\dot{V}_{1}(t)=-k_{1}\left|s_{1}\right| \text {. }
$$

Step 2. Choose the second control Lyapunov function

$$
V_{2}(t)=\frac{1}{2} s_{2}^{2}
$$

By taking its derivative with respect to time yields

$$
\dot{V}_{2}(t)=s_{2} \dot{s}_{2}=s_{2}\left(D^{q_{2}} e_{2}+\lambda_{2} e_{2}\right) .
$$

Substituting the second state equation of (17) into (28), one has

$$
\begin{gathered}
\dot{V}_{2}(t)=s_{2}\left[(28-35 \alpha) e_{1}+e_{1} e_{3}-e_{1} y_{3}-e_{3} y_{1}\right. \\
\left.+(29 \alpha-1) e_{2}+u_{2}+\lambda_{2} e_{2}\right] .
\end{gathered}
$$

Therefore, by designing the second control law as

$$
\begin{aligned}
u_{2}= & -(28-35 \alpha) e_{1}-e_{1} e_{3}+e_{1} y_{3}+e_{3} y_{1} \\
& -(29 \alpha-1) e_{2}-\lambda_{2} e_{2}-k_{2} \operatorname{sgn}\left(s_{3}\right),
\end{aligned}
$$

where $k_{2}>0$.

Equation (29) becomes

$$
\dot{V}_{2}(t)=-k_{2}\left|s_{2}\right| .
$$

Step 3. Choose the third control Lyapunov function

$$
V_{3}(t)=\frac{1}{2} s_{3}^{2}
$$

Its time derivative is given by

$$
\dot{V}_{3}(t)=s_{3} \dot{s}_{3}=s_{3}\left(D^{q_{3}} e_{3}+\lambda_{3} e_{3}\right) .
$$

Substituting the third state equation of (17) into (33), one has

$$
\dot{V}_{3}(t)=s_{3}\left[-e_{1} e_{2}+e_{1} y_{2}+e_{2} y_{1}-\frac{(8+\alpha) e_{3}}{3}+u_{3}+\lambda_{3} e_{3}\right] \text {. }
$$

We are, then, in the position to design the third control law as follows:

$$
u_{3}=e_{1} e_{2}-e_{1} y_{2}-e_{2} y_{1}+\frac{(8+\alpha) e_{3}}{3}-\lambda_{3} e_{3}-k_{3} \operatorname{sgn}\left(s_{3}\right),
$$

where $k_{3}>0$.

With this choice, (34) can be rewritten as

$$
\dot{V}_{3}(t)=-k_{3}\left|s_{3}\right| \text {. }
$$

Step 4. Finally, we gather the above three control functions as

$$
V\left(s_{1}, s_{2}, s_{3}\right)=\frac{1}{2} s_{1}^{2}+\frac{1}{2} s_{2}^{2}+\frac{1}{2} s_{3}^{2} .
$$

It is clear from (26), (31), and (36) that

$$
\dot{V}\left(s_{1}, s_{2}, s_{3}\right)=-\left(k_{1}\left|s_{1}\right|+k_{2}\left|s_{2}\right|+k_{3}\left|s_{3}\right|\right) .
$$

There exists some $k>0$ such that

$$
k_{1}\left|s_{1}\right|+k_{2}\left|s_{2}\right|+k_{3}\left|s_{3}\right|>k\|s\|
$$

where

$$
\|s\|=\sqrt{s_{1}^{2}+s_{2}^{2}+s_{3}^{2}}
$$

The resulting derivative of $V$ is

$$
\dot{V}\left(s_{1}, s_{2}, s_{3}\right)<-k\|s\|
$$

In terms of Lemma 8, the Lyapunov function (37) provides the proof of globally asymptotical stability with the control laws (24), (30), and (35).

4.2. Synchronization of the Uncertain System. In this subsection, we will proceed to study the synchronization of the fractional-order unified chaotic system in the presence of systematic uncertainties and external disturbances which can be hardly ignored in the real-world application. It is assumed that systematic uncertainties $\Delta f_{1}, \Delta f_{2}$, and $\Delta f_{3}$ and external disturbances $d_{1}, d_{2}$, and $d_{3}$ are all bounded; that is, $\left|\Delta f_{i}\right|<$ $\rho_{i}$ and $\left|d_{i}(t)\right|<\theta_{i}$, where $\rho_{i}$ and $\theta_{i}$ are unknown positive constants, $i=1,2,3$. Let us denote that $\hat{\rho}_{i}$ is an estimate of $\rho_{i}$, while $\widehat{\theta}_{i}$ is an estimate of $\theta_{i}$. Since $\rho_{i}$ and $\theta_{i}$ are unknown, our task in this subsection is fulfilled with an adaptive controller consisting of control laws and update laws to obtain $\widehat{\rho}_{i}$ and $\widehat{\theta}_{i}$, $i=1,2,3$.

The uncertain fractional-order unified chaotic system can be described as

$$
\begin{gathered}
D^{q_{1}} e_{1}=(25 \alpha+10)\left(e_{2}-e_{1}\right)+\Delta f_{1}+d_{1}+u_{1}, \\
D^{q_{2}} e_{2}=(28-35 \alpha) e_{1}+e_{1} e_{3}-e_{1} y_{3}-e_{3} y_{1} \\
+(29 \alpha-1) e_{2}+\Delta f_{2}+d_{2}+u_{2}, \\
D^{q_{3}} e_{3}=-e_{1} e_{2}+e_{1} y_{2}+e_{2} y_{1}-\frac{(8+\alpha) e_{3}}{3}+\Delta f_{3}+d_{3}+u_{3} .
\end{gathered}
$$

The adaptive sliding mode design of system (42) consists of four steps which are elaborated as follows.

Step 1. Consider the first control Lyapunov function

$$
V_{1}(t)=\frac{1}{2}\left[s_{1}^{2}+\frac{1}{\mu_{1}}\left(\hat{\rho}_{1}-\rho_{1}\right)^{2}+\frac{1}{\gamma_{1}}\left(\widehat{\theta}_{1}-\theta_{1}\right)^{2}\right],
$$


the time derivative of which becomes

$$
\begin{aligned}
\dot{V}_{1}(t)= & s_{1} \dot{s}_{1}+\frac{1}{\mu_{1}}\left(\hat{\rho}_{1}-\rho_{1}\right) \dot{\hat{\rho}}_{1}+\frac{1}{\gamma_{1}}\left(\widehat{\theta}_{1}-\theta_{1}\right) \dot{\hat{\theta}}_{1} \\
= & s_{1}\left(D^{q_{1}} e_{1}+\lambda_{1} e_{1}\right)+\frac{1}{\mu_{1}}\left(\widehat{\rho}_{1}-\rho_{1}\right) \dot{\hat{\rho}}_{1} \\
& +\frac{1}{\gamma_{1}}\left(\hat{\theta}_{1}-\theta_{1}\right) \dot{\hat{\theta}}_{1} .
\end{aligned}
$$

Substituting the first state equation of (17) into (44), one has

$$
\begin{aligned}
\dot{V}_{1}(t)= & s_{1}\left[(25 \alpha+10)\left(e_{2}-e_{1}\right)+\Delta f_{1}+d_{1}+u_{1}+\lambda_{1} e_{1}\right] \\
& +\frac{1}{\mu_{1}}\left(\widehat{\rho}_{1}-\rho_{1}\right) \dot{\hat{\rho}}_{1}+\frac{1}{\gamma_{1}}\left(\widehat{\theta}_{1}-\theta_{1}\right) \dot{\hat{\theta}}_{1} .
\end{aligned}
$$

By designing the first control law and adaptive law as

$$
\begin{gathered}
u_{1}=-(25 \alpha+10)\left(e_{2}-e_{1}\right)-\lambda_{1} e_{1}-\left(\widehat{\rho}_{1}+\widehat{\theta}_{1}+k_{1}\right) \operatorname{sgn}\left(s_{1}\right), \\
\dot{\hat{\rho}}_{1}=\mu_{1}\left|s_{1}\right| \\
\dot{\hat{\theta}}_{1}=\gamma_{1}\left|s_{1}\right|
\end{gathered}
$$

then, (45) becomes

$$
\begin{aligned}
\dot{V}_{1}= & s\left[\left(\Delta f_{1}+d_{1}\right)-\left(\hat{\rho}_{1}+\widehat{\theta}_{1}+k_{1}\right) \operatorname{sgn}\left(s_{1}\right)\right] \\
& +\left(\widehat{\rho}_{1}-\rho_{1}\right)\left|s_{1}\right|+\left(\widehat{\theta}_{1}-\theta_{1}\right)\left|s_{1}\right| \\
\leq & \left(\left|\Delta f_{1}\right|+\left|d_{1}\right|\right)\left|s_{1}\right|-\left(\hat{\rho}_{1}+\widehat{\theta}_{1}+k_{1}\right)\left|s_{1}\right| \\
& +\left(\widehat{\rho}_{1}-\rho_{1}\right)\left|s_{1}\right|+\left(\widehat{\theta}_{1}-\theta_{1}\right)\left|s_{1}\right| \\
< & \left(\rho_{1}+\theta_{1}\right)\left|s_{1}\right|-\left(\hat{\rho}_{1}+\widehat{\theta}_{1}+k_{1}\right)\left|s_{1}\right| \\
& +\left(\hat{\rho}_{1}-\rho_{1}\right)\left|s_{1}\right|+\left(\widehat{\theta}_{1}-\theta_{1}\right)\left|s_{1}\right|=-k_{1}\left|s_{1}\right| .
\end{aligned}
$$

Step 2. Choose the second control Lyapunov function

$$
V_{2}(t)=\frac{1}{2}\left[s_{2}^{2}+\frac{1}{\mu_{2}}\left(\hat{\rho}_{2}-\rho_{2}\right)^{2}+\frac{1}{\gamma_{2}}\left(\widehat{\theta}_{2}-\theta_{2}\right)^{2}\right]
$$

whose derivative is

$$
\begin{aligned}
\dot{V}_{2}(t)= & s_{2} \dot{s}_{2}+\frac{1}{\mu_{2}}\left(\widehat{\rho}_{2}-\rho_{2}\right) \dot{\hat{\rho}}_{2}+\frac{1}{\gamma_{2}}\left(\widehat{\theta}_{2}-\theta_{2}\right) \dot{\hat{\theta}}_{2} \\
= & s_{2}\left(D^{q_{2}} e_{2}+\lambda_{2} e_{2}\right)+\frac{1}{\mu_{2}}\left(\widehat{\rho}_{2}-\rho_{2}\right) \dot{\hat{\rho}}_{2} \\
& +\frac{1}{\gamma_{1}}\left(\hat{\theta}_{2}-\theta_{2}\right) \dot{\hat{\theta}}_{2} .
\end{aligned}
$$

Substituting the second state equation of (17) into (50), one has

$$
\begin{aligned}
\dot{V}_{2}(t)= & s_{2}[ \\
& \left.-e_{3} y_{1}+(28 \alpha-35) e_{1}+e_{1} e_{3}-e_{1} y_{3}\right] \\
+ & s_{2}\left[\Delta f_{2}+d_{2}+u_{2}+\lambda_{2} e_{2}\right] \\
+ & \frac{1}{\mu_{2}}\left(\hat{\rho}_{2}-\rho_{2}\right) \dot{\hat{\rho}}_{2}+\frac{1}{\gamma_{2}}\left(\hat{\theta}_{2}-\theta_{2}\right) \dot{\hat{\theta}}_{2} .
\end{aligned}
$$

We choose the control law and the adaptive law

$$
\begin{gathered}
u_{2}=-(28-35 \alpha) e_{1}-e_{1} e_{3}+e_{1} y_{3}+e_{3} y_{1} \\
-(29 \alpha-1) e_{2}-\lambda_{2} e_{2}-\left(\hat{\rho}_{2}+\hat{\theta}_{2}+k_{2}\right) \operatorname{sgn}\left(s_{2}\right), \\
\dot{\hat{\rho}}_{2}=\mu_{2}\left|s_{2}\right|, \\
\dot{\hat{\theta}}_{2}=\gamma_{2}\left|s_{2}\right| .
\end{gathered}
$$

With this choice, (51) becomes

$$
\dot{V}_{2}(t)<-k_{2}\left|s_{2}\right| .
$$

Step 3. Choose the third Lyapunov function

$$
V_{3}(t)=\frac{1}{2}\left[s_{3}^{2}+\frac{1}{\mu_{3}}\left(\hat{\rho}_{3}-\rho_{3}\right)^{2}+\frac{1}{\gamma_{3}}\left(\widehat{\theta}_{3}-\theta_{3}\right)^{2}\right] .
$$

Taking time derivative gives

$$
\begin{aligned}
\dot{V}_{3}(t)= & s_{3} \dot{s}_{3}+\frac{1}{\mu_{3}}\left(\hat{\rho}_{3}-\rho_{3}\right) \dot{\hat{\rho}}_{3}+\frac{1}{\gamma_{3}}\left(\widehat{\theta}_{3}-\theta_{3}\right) \dot{\hat{\theta}}_{3} \\
= & s_{3}\left(D^{q_{3}} e_{3}+\lambda_{3} e_{3}\right)+\frac{1}{\mu_{3}}\left(\hat{\rho}_{3}-\rho_{3}\right) \dot{\hat{\rho}}_{3} \\
& +\frac{1}{\gamma_{3}}\left(\widehat{\theta}_{3}-\theta_{3}\right) \dot{\hat{\theta}}_{3} .
\end{aligned}
$$

Substituting the third state equation of (17) into (57), one has

$$
\begin{aligned}
\dot{V}_{3}(t)= & s_{3}\left[-e_{1} e_{2}+e_{1} y_{2}+e_{2} y_{1}-\frac{(8+\alpha) e_{3}}{3}\right] \\
& +s_{3}\left[\Delta f_{3}+d_{3}+u_{3}+\lambda_{3} e_{3}\right] \\
& +\frac{1}{\mu_{3}}\left(\hat{\rho}_{3}-\rho_{3}\right) \dot{\hat{\rho}}_{3}+\frac{1}{\gamma_{3}}\left(\widehat{\theta}_{3}-\theta_{3}\right) \dot{\hat{\theta}}_{3} .
\end{aligned}
$$

We choose the third control law and adaptive law

$$
\begin{gathered}
u_{3}=e_{1} e_{2}-e_{1} y_{2}-e_{2} y_{1}+\frac{(8+\alpha) e_{3}}{3}-\lambda_{3} e_{3} \\
-\left(\hat{\rho}_{3}+\widehat{\theta}_{3}+k_{3}\right) \operatorname{sgn}\left(s_{3}\right), \\
\dot{\hat{\rho}}_{3}=\mu_{3}\left|s_{3}\right|, \\
\dot{\hat{\theta}}_{3}=\gamma_{3}\left|s_{3}\right| .
\end{gathered}
$$

Then, the resulting derivative of $V_{3}$ is

$$
\dot{V}_{3}(t)<-k_{3}\left|s_{3}\right| \text {. }
$$




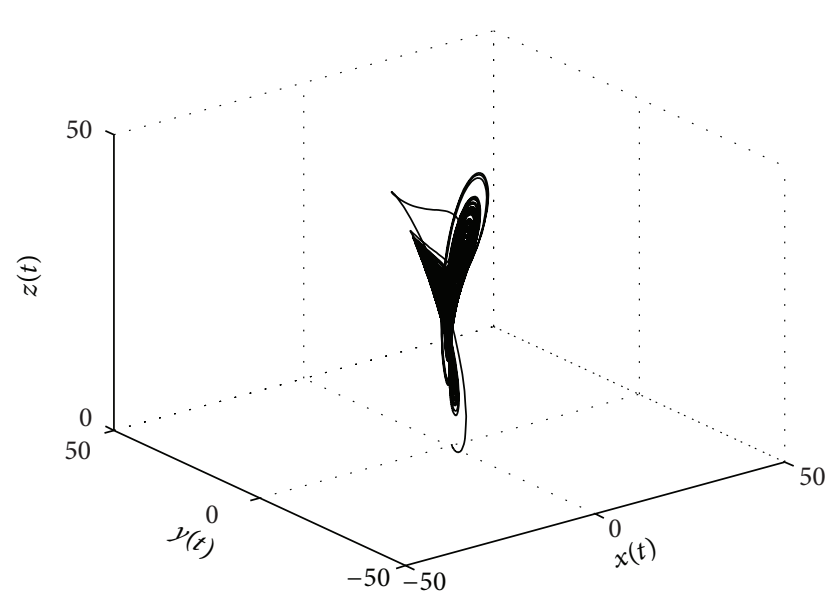

(a)

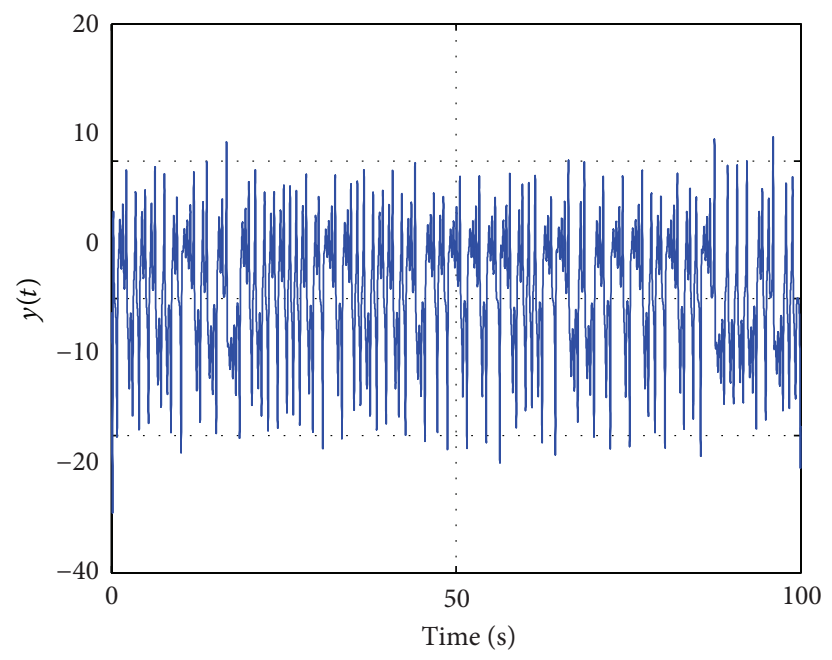

(c)

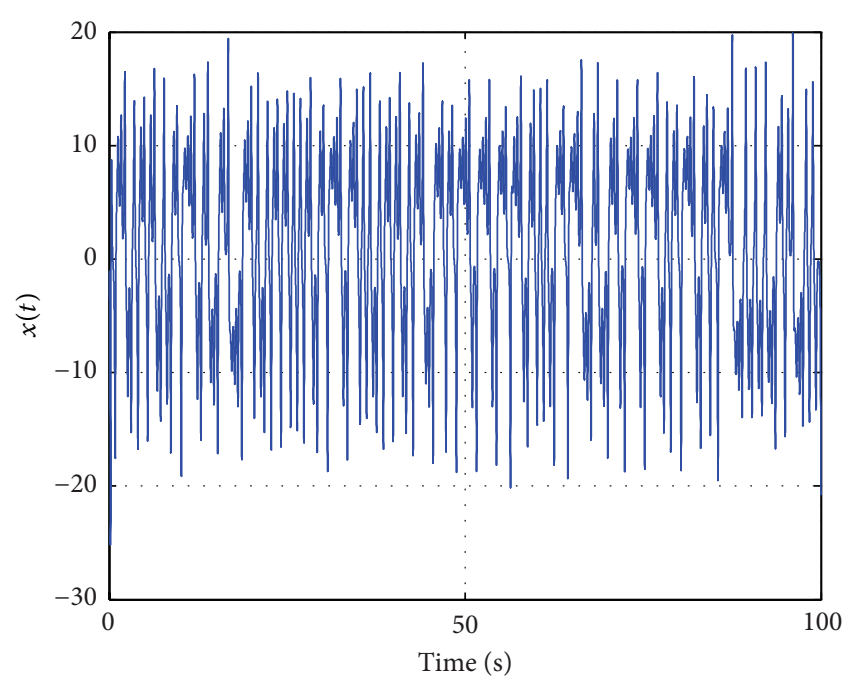

(b)

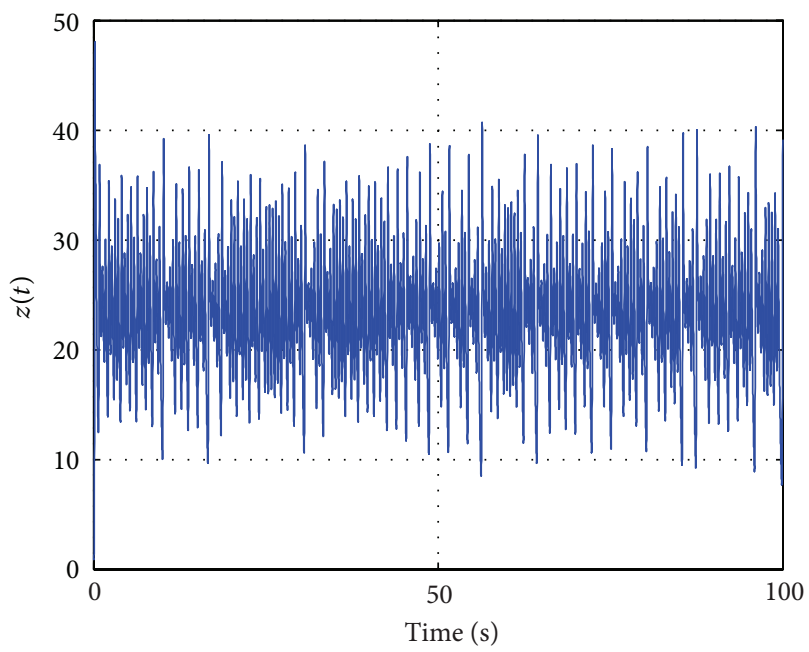

(d)

Figure 1: Chaotic trajectories with $\alpha=0.5$.

Step 4. Finally, we gather the above three control functions as

$$
V(t)=\frac{1}{2} \sum_{i=1}^{3} s_{i}^{2}+\sum_{i=1}^{3} \frac{1}{2 \mu_{i}}\left(\widehat{\rho}_{i}-\rho_{i}\right)^{2}+\sum_{i=1}^{3} \frac{1}{2 \gamma_{i}}\left(\widehat{\theta}_{i}-\theta_{i}\right)^{2} .
$$

It is clear from (48), (55), and (61) that

$$
\dot{V}(t)=-\left(k_{1}\left|s_{1}\right|+k_{2}\left|s_{2}\right|+k_{3}\left|s_{3}\right|\right) .
$$

There exists some $k>0$ such that

$$
k_{1}\left|s_{1}\right|+k_{2}\left|s_{2}\right|+k_{3}\left|s_{3}\right|>k\|s\| .
$$

The resulting derivative of $\dot{V}$ is

$$
\dot{V}\left(s_{1}, s_{2}, s_{3}\right)<-k\|s\|,
$$

where

$$
\|s\|=\sqrt{s_{1}^{2}+s_{2}^{2}+s_{3}^{2}} .
$$

By using Lemma 8 , this Lyapunov function provides the proof of globally asymptotical stability with the control laws (46), (52), and (59) and adaptive laws (47), (53), and (60).

\section{Numerical Simulations}

5.1. Chaotic Behaviors of Fractional-Order Chaotic System. In [55], the authors have provided us with numerical methods of fractional calculus. In [56], the chaotic behaviors of the fractional-order unified system were numerically investigated, where it is found that the lowest order to exhibit chaos is 2.76 .

The chaotic behaviors are presented in Figures 1 and 2 with fractional orders of $q_{1}=0.93, q_{2}=0.94$, and 


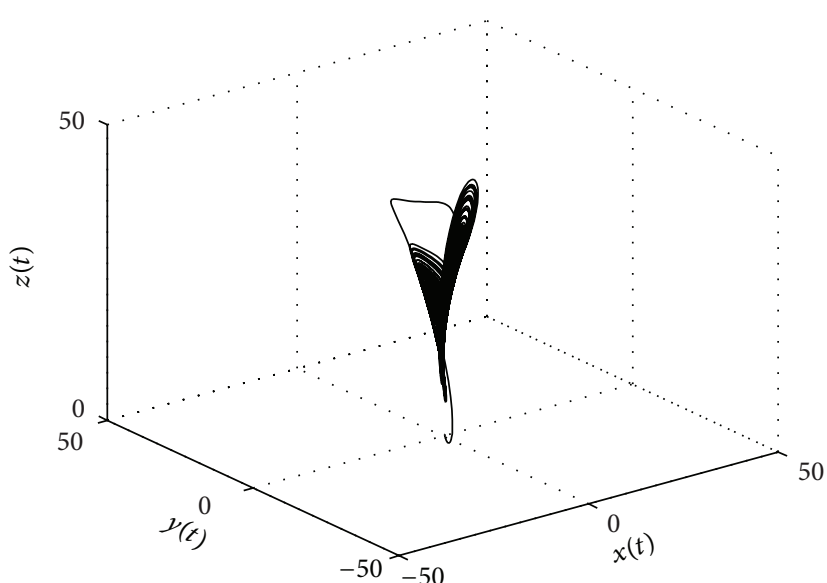

(a)

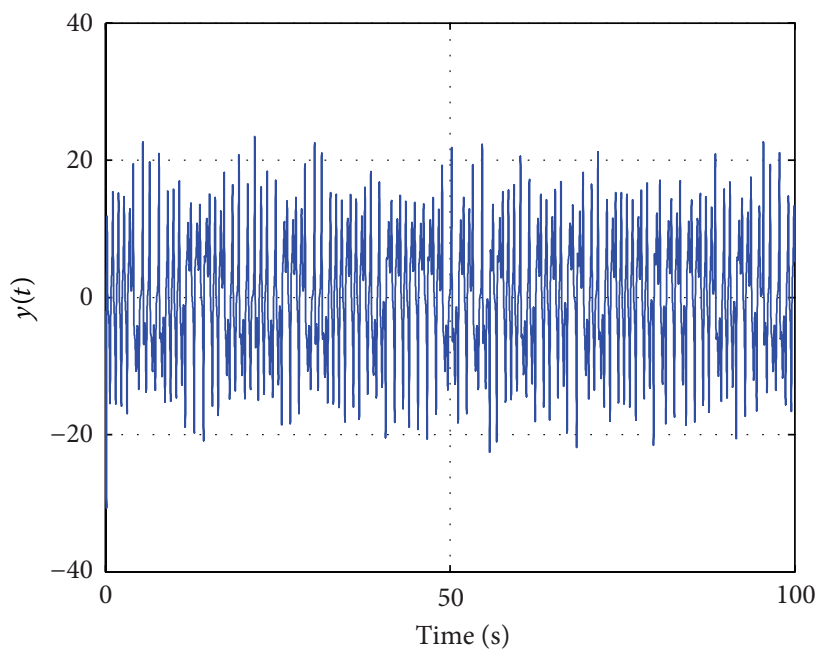

(c)

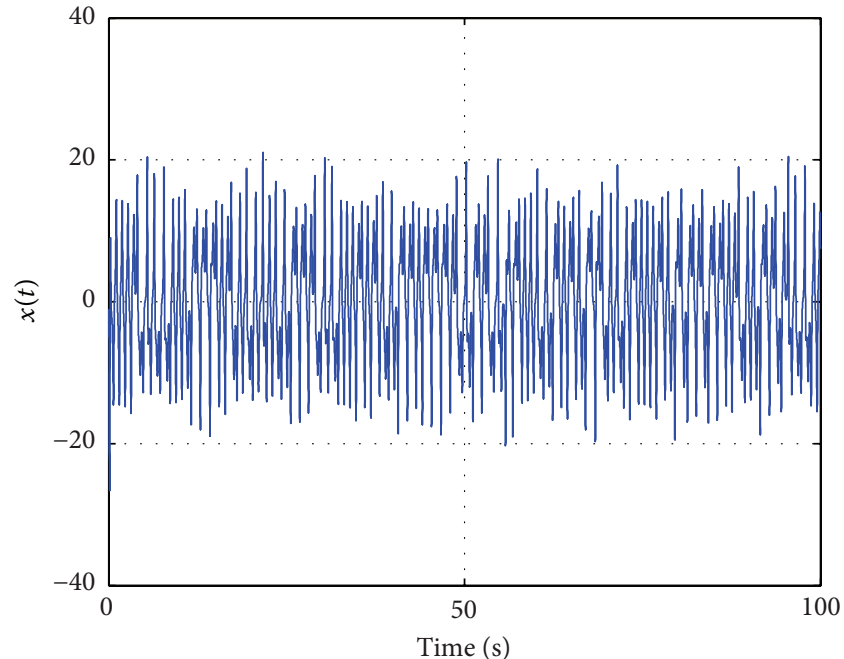

(b)

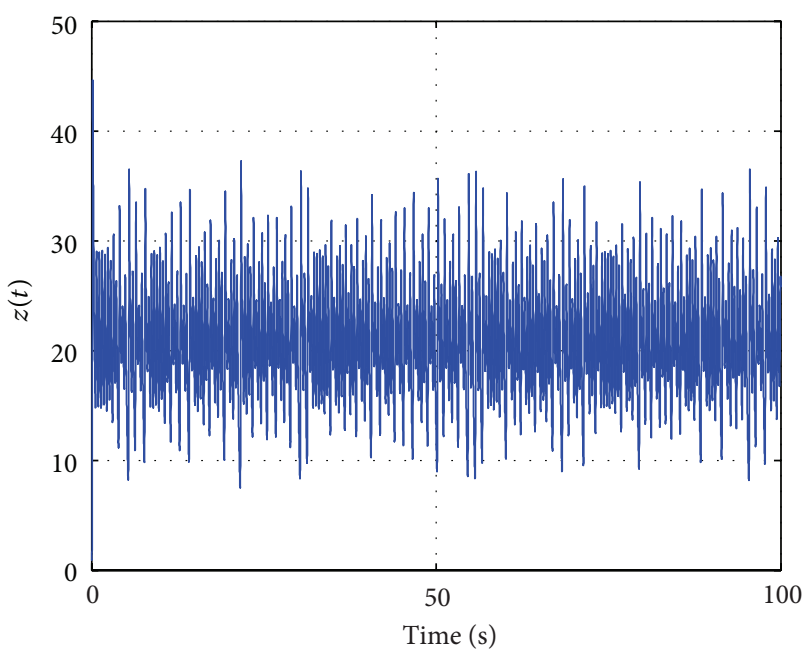

(d)

FIGURE 2: Chaotic trajectories with $\alpha=1$.

$q_{3}=0.95$ and the initial conditions of $\left(x_{1}(0), x_{2}(0), x_{3}(0)\right)=$ $(-1,-2,1)$. The numerical algorithm is based on the following Grünwald-Letnikov's definition:

$$
\begin{aligned}
x\left(t_{k}\right)= & (25 \alpha+10)\left(x_{2}\left(t_{k-1}\right)-x_{1}\left(t_{k-1}\right)\right) h^{q_{1}} \\
& -\sum_{j=2}^{k} c_{j}^{\left(q_{1}\right)} x_{1}\left(t_{k-j}\right), \\
x_{2}\left(t_{k}\right)= & {\left[(28-35 \alpha) x_{1}\left(t_{k}\right)-x_{1}\left(t_{k}\right) x_{3}\left(t_{k-1}\right)\right.} \\
& \left.+(29 \alpha-1) x_{2}\left(t_{k-1}\right)\right] h^{q_{2}}-\sum_{j=2}^{k} c_{j}^{\left(q_{2}\right)} x_{2}\left(t_{k-j}\right), \\
x_{3}\left(t_{k}\right)= & {\left[x_{1}\left(t_{k}\right) x_{2}\left(t_{k}\right)-\frac{\alpha+8}{3} x_{3}\left(t_{k-1}\right)\right] h^{q_{3}} } \\
& -\sum_{j=2}^{k} c_{j}^{\left(q_{3}\right)} x_{3}\left(t_{k-j}\right),
\end{aligned}
$$

where $T_{\text {sim }}$ is the simulation time, $k=1,2, \ldots, N$, for $N=$ $\left[T_{\text {sim }} / h\right]$.

5.2. Simulations of Synchronization of the Nominal System. In this subsection, numerical simulations are presented to demonstrate the effectiveness of the proposed sliding model control in Section 4.1. In the numerical simulations, the fractional orders are chosen as $q_{1}=0.93, q_{2}=0.94$, and $q_{3}=0.95$. The initial conditions of the drive system (15) and the response system (16) are chosen as $\left(x_{1}(0), x_{2}(0), x_{3}(0)\right)=$ $(-1,-2,1)$ and $\left(y_{1}(0), y_{2}(0), y_{3}(0)\right)=(4,-4,4)$, respectively. Parameters in (18) are chosen as $\lambda_{1}=\lambda_{2}=\lambda_{3}=4$. Gains of the control inputs in (24), (30), and (35) are chosen as $k_{1}=k_{2}=k_{2}=1$.

When $\alpha=0.5$ and $\alpha=1$, numerical simulations of synchronization of system (15) are presented in Figures 3, $4,5,6,7$, and 8 with control inputs (24), (30), and (35). For interpretations of the references to colors in these figure legends, the reader is referred to the web version of this paper. 


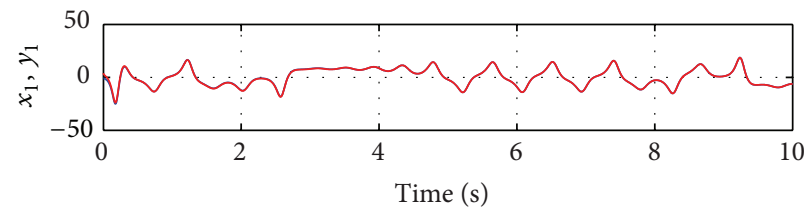

(a)

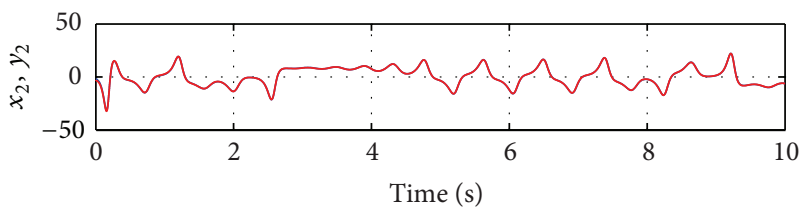

(b)

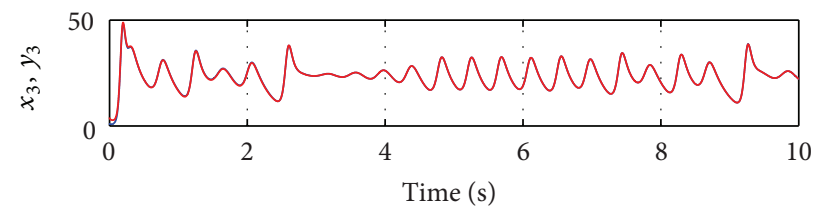

(c)

FIGURE 3: Synchronization of determined fractional-order unified chaotic system with $\alpha=0.5$ (blue line represents the trajectories of the drive system, while red line represents the trajectories of the response system).

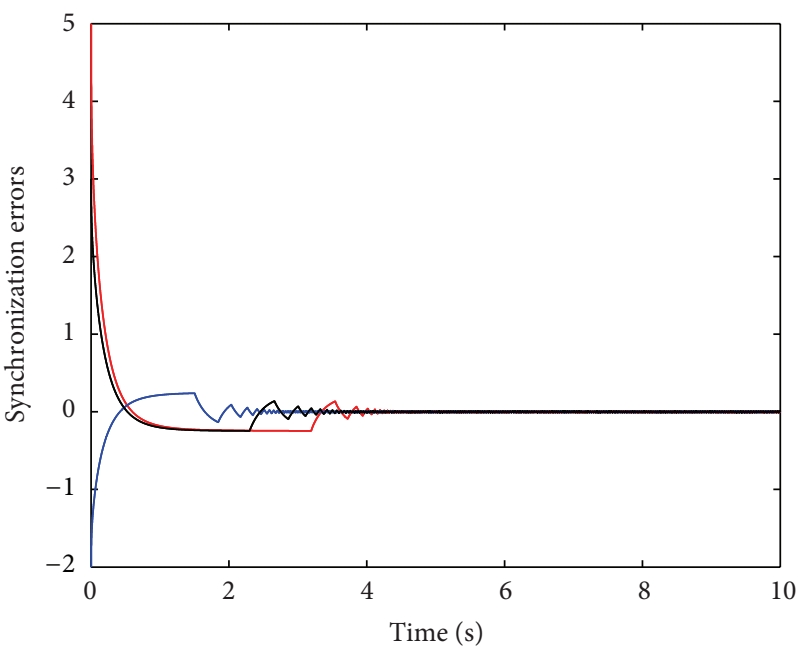

FIGURE 4: Synchronization errors states with $\alpha=0.5$ (red line represents the first error state $e_{1}$, blue line represents the second error state $e_{2}$, and black line represents the third error state $e_{3}$ ).

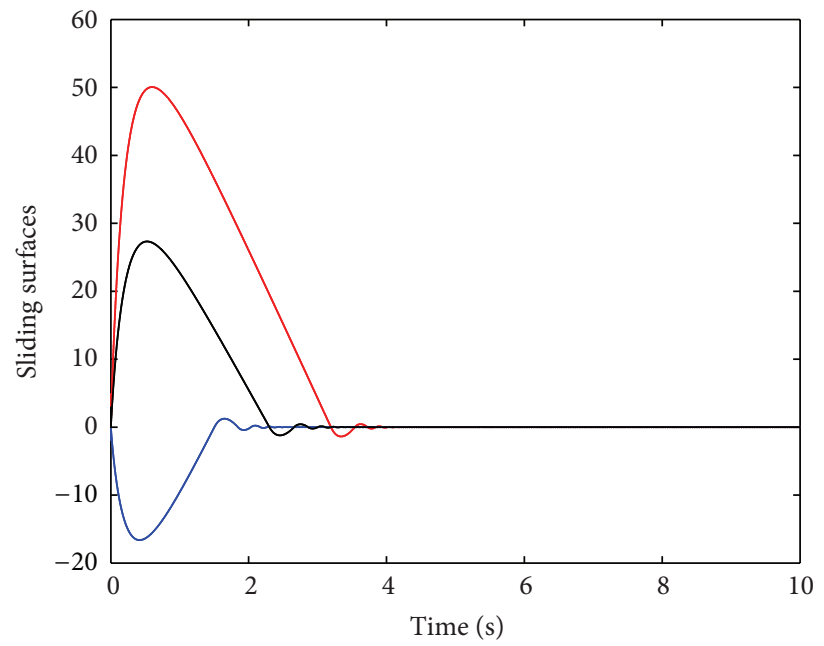

FIGURE 5: Sliding surfaces states with $\alpha=0.5$ (red line represents the first sliding surface state $s_{1}$, blue line represents the second sliding surface state $s_{2}$, and black line represents the third sliding surface state $s_{3}$ ). 


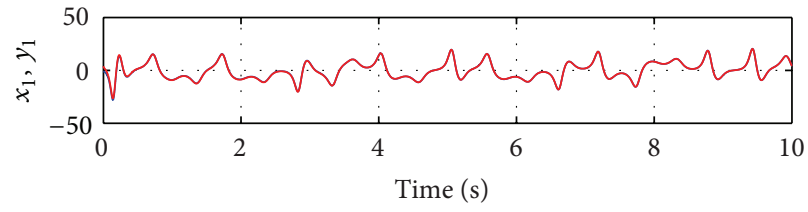

(a)

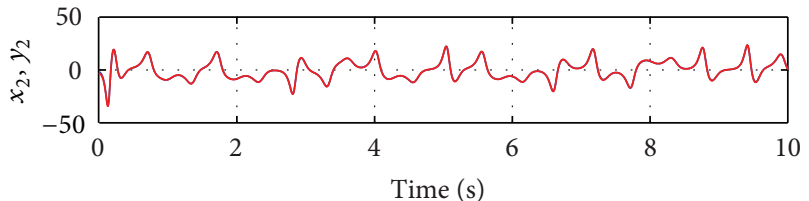

(b)

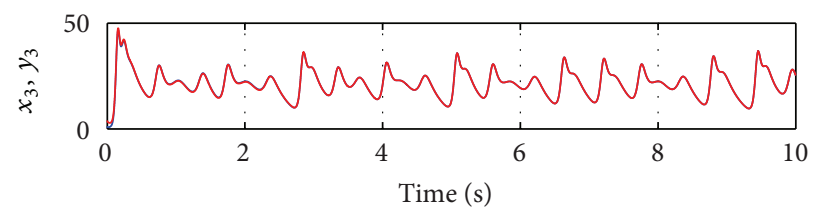

(c)

FIGURE 6: Synchronization of determined fractional-order unified chaotic system with $\alpha=1$ (blue line represents the trajectories of the drive system, while red line represents the trajectories of the response system).

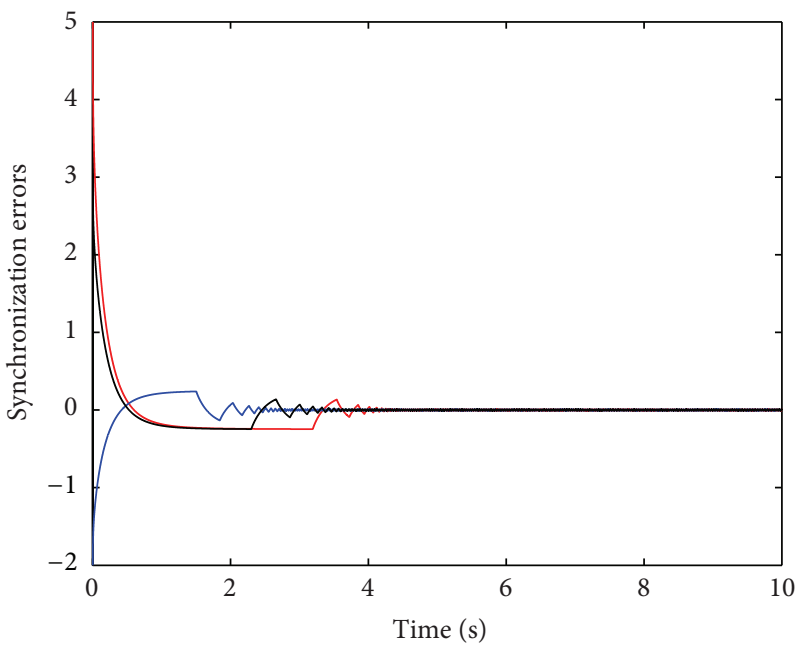

FIGURE 7: Synchronization errors states with $\alpha=1$ (red line represents the first error state $e_{1}$, blue line represents the second error state $e_{2}$, and black line represents the third error state $e_{3}$ ).

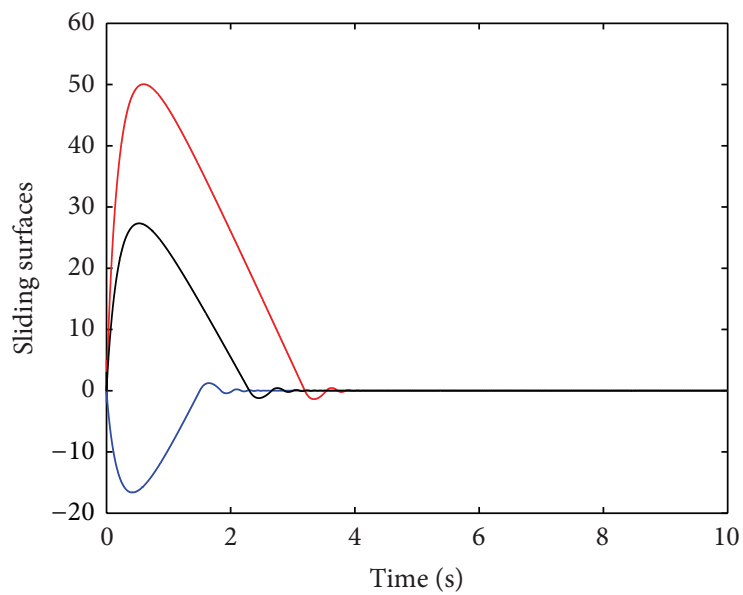

FIGURE 8: Sliding surfaces states with $\alpha=1$ (red line represents the first sliding surface state $s_{1}$, blue line represents the second sliding surface state $s_{2}$, and black line represents the third sliding surface state $s_{3}$ ). 


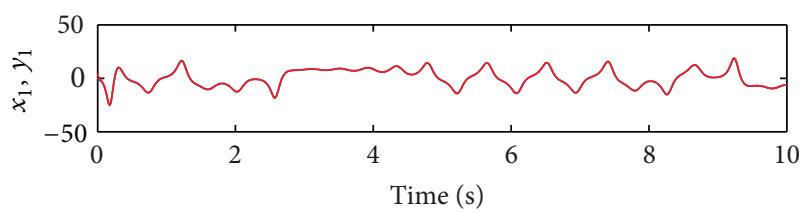

(a)

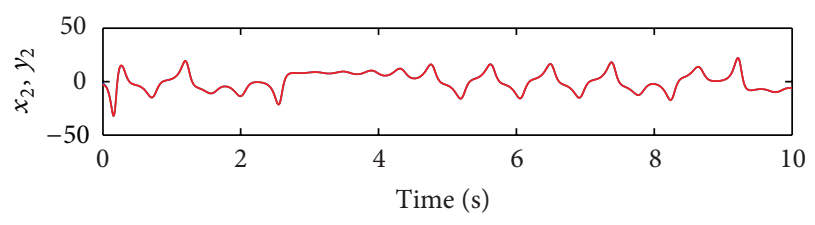

(b)

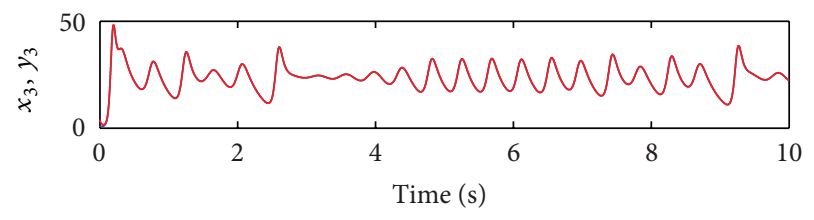

(c)

FIGURE 9: Synchronization of fractional-order unified chaotic system in the presence of systematic uncertainties and external disturbances with $\alpha=0.5$ (blue line represents the trajectories of the drive system, while red line represents the trajectories of the response system).

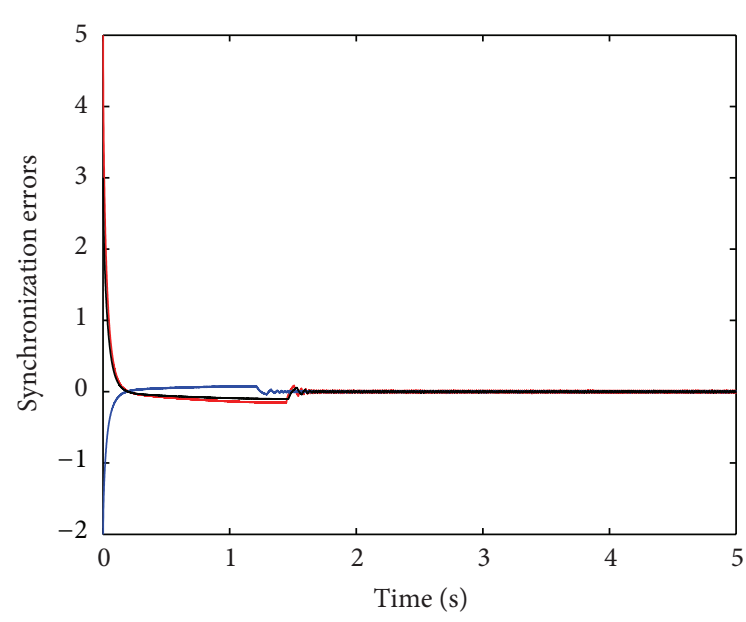

FIGURE 10: Synchronization errors states in the presence of systematic uncertainties and external disturbances with $\alpha=0.5$ (red line represents the first error state $e_{1}$, blue line represents the second error state $e_{2}$, and black line represents the third error state $e_{3}$ ).

5.3. Simulations of Synchronization of the Uncertain System. In this subsection, numerical simulations are presented to demonstrate the effectiveness of the proposed adaptive sliding model control in Section 4.2. In the numerical simulations, the fractional orders are always chosen as $q_{1}=0.93$, $q_{2}=0.94$, and $q_{3}=0.95$. The initial conditions of the drive system (15) and the response system (16) are also chosen as $\left(x_{1}(0), x_{2}(0), x_{3}(0)\right)=(-1,-2,1)$ and $\left(y_{1}(0), y_{2}(0), y_{3}(0)\right)=$ $(4,-4,4)$, respectively. Parameters in (18) are chosen as

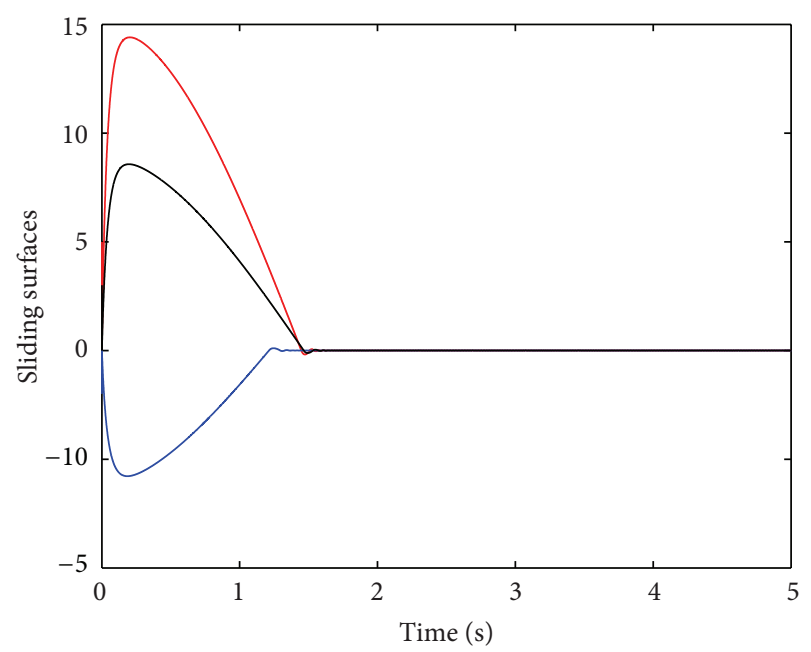

FIGURE 11: Sliding surfaces states in the presence of systematic uncertainties and external disturbances with $\alpha=0.5$ (red line represents the first sliding surface state $s_{1}$, blue line represents the second sliding surface state $s_{2}$, and black line represents the third sliding surface state $s_{3}$ ).

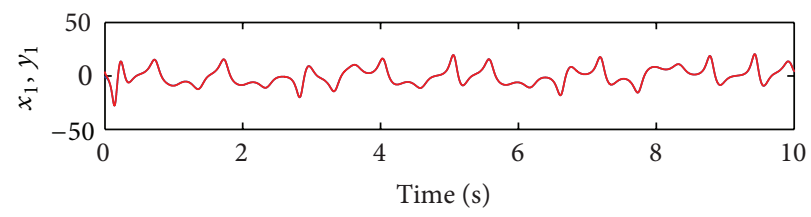

(a)

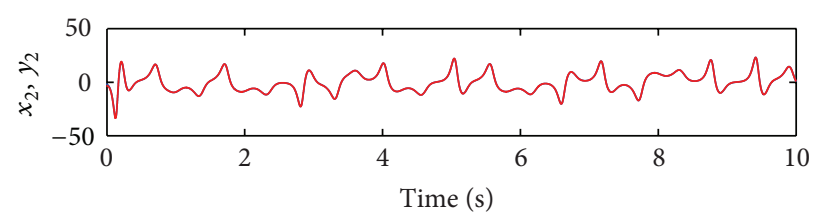

(b)

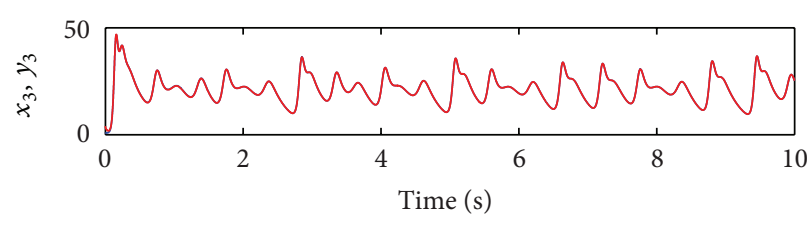

(c)

FIGURE 12: Synchronization of fractional-order unified chaotic system in the presence of systematic uncertainties and external disturbances with $\alpha=1$ (blue line represents the trajectories of the drive system, while red line represents the trajectories of the response system).

$\lambda_{1}=\lambda_{2}=\lambda_{3}=20$. Gains of the control laws (46), (52), and (59) are chosen as $k_{1}=k_{2}=k_{2}=0.5$. Gains of the adaptive laws (47), (53), and (60) are chosen as $\mu_{1}=\gamma_{1}=$ $\mu_{2}=\gamma_{2}=\mu_{3}=\gamma_{3}=0.1$. Systematic uncertainties and external disturbances are assumed to be $\Delta f_{1}=0, d_{1}=$ $-3 \cos (\pi(t-0.1)), \Delta f_{2}=-0.6 \sin \left(2\left(y_{1}-x_{1}\right)\right), d_{2}=5 \sin (t)$, $\Delta f_{3}=0$, and $d_{3}=2 \cos (t-0.1)$. 


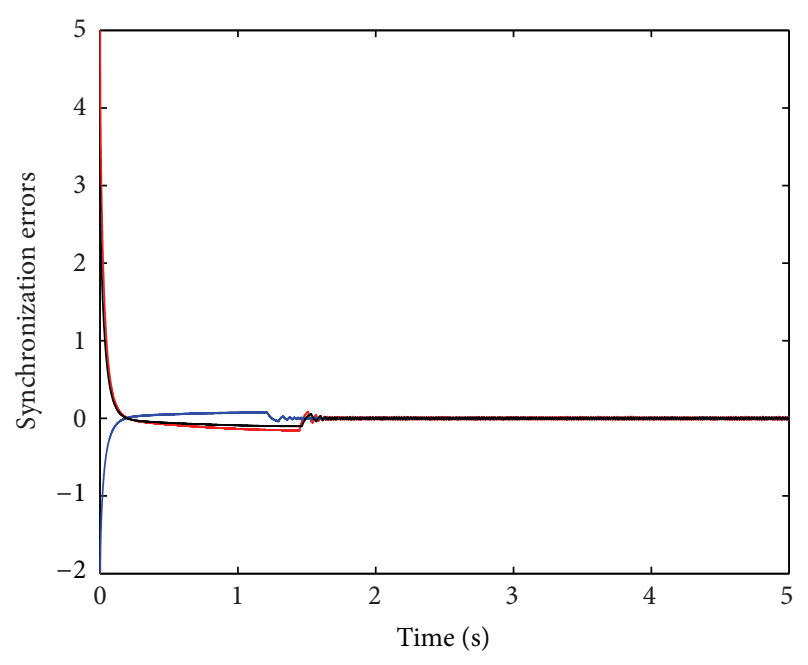

FIGURE 13: Synchronization errors states in the presence of systematic uncertainties and external disturbances with $\alpha=1$ (red line represents the first error state $e_{1}$, blue line represents the second error state $e_{2}$, and black line represents the third error state $e_{3}$ ).

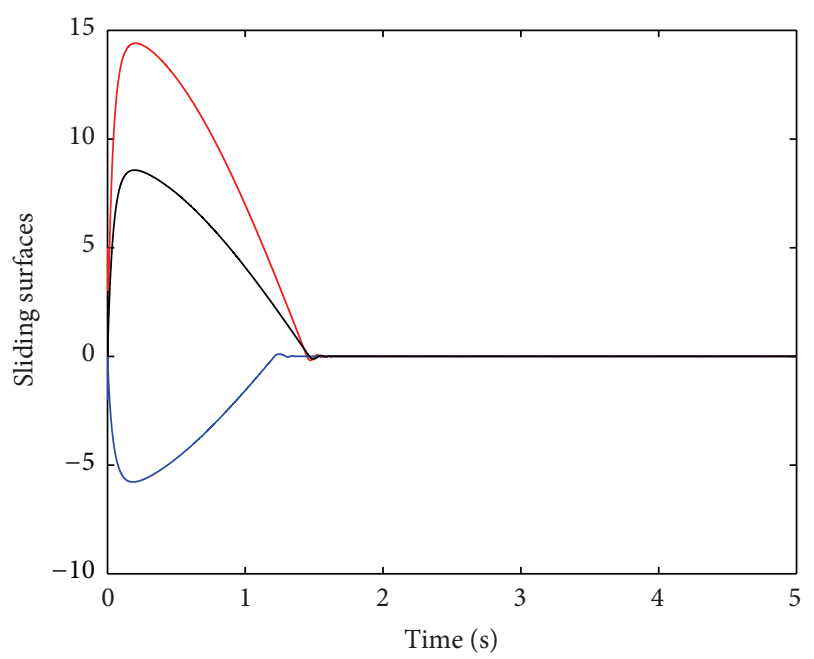

FIGURE 14: Sliding surfaces states in the presence of systematic uncertainties and external disturbances with $\alpha=1$ (red line represents the first sliding surface state $s_{1}$, blue line represents the second sliding surface state $s_{2}$, and black line represents the third sliding surface state $s_{3}$ ).

When $\alpha=0.5$ and $\alpha=1$, numerical simulations are presented in Figures 9, 10,11, 12, 13, and 14 with control inputs (46), (52), and (59) and adaptive laws (47), (53), and (60). For interpretations of the references to colors in these figure legends, the reader is referred to the web version of this paper.

\section{Conclusions}

This work is concerned with robust synchronization of the fractional-order unified chaotic system. The sliding mode control technique was applied to propose the control design of nominal system and adaptive sliding mode control scheme was designed to develop the control laws and adaptive laws for uncertain system with systematic uncertainties and external disturbances whose bounds are unknown. Numerical simulations were presented to demonstrate the effectiveness of the two kinds of techniques.

\section{References}

[1] K. Diethelm, The Analysis of Fractional Differential Equations: An Application-Oriented Exposition Using Differential Operators of Caputo Type, vol. 2004, Springer, Berlin, Germany, 2010.

[2] I. Podlubny, Fractional Differential Equations: An Introduction to Fractional Derivatives, Fractional Differential Equations, to Methods of Their Solution and Some of Their Applications, vol. 198, Academic Press, San Diego, Calif, USA, 1999.

[3] Y. Chen and I. Petras, "Fractional order control: a tutorial," in Proceedings of the American Control Conference Hyatt Regency Riverfront, St. Louis, MO, USA, 2009.

[4] J. T. Machado, V. Kiryakova, and F. Mainardi, "Recent history of fractional calculus," Communications in Nonlinear Science and Numerical Simulation, vol. 16, no. 3, pp. 1140-1153, 2011.

[5] C. P. Li and F. R. Zhang, "A survey on the stability of fractional differential equations," European Physical Journal, vol. 193, no. 1, pp. 27-47, 2011.

[6] Y. H. Wang, "Mittag-Leffler stability of a hybrid ratio-dependent fractional three species food chain," Communications in Fractional Calculus, vol. 3, pp. 111-118, 2012.

[7] T. T. Hartley, C. F. Lorenzo, and H. K. Qammer, "Chaos in a fractional order Chua's system," IEEE Transactions on Circuits and Systems I, vol. 42, no. 8, pp. 485-490, 1995.

[8] I. Petráš, "A note on the fractional-order Chua’s system," Chaos, Solitons and Fractals, vol. 38, no. 1, pp. 140-147, 2008.

[9] J.-H. Chen and W.-C. Chen, "Chaotic dynamics of the fractionally damped van der Pol equation," Chaos, Solitons and Fractals, vol. 35, no. 1, pp. 188-198, 2008.

[10] R. S. Barbosa, J. A. T. MacHado, B. M. Vinagre, and A. J. Caldern, "Analysis of the Van der Pol oscillator containing derivatives of fractional order," Journal of Vibration and Control, vol. 13, no. 9-10, pp. 1291-1301, 2007.

[11] M. S. Tavazoei, M. Haeri, M. Attari, S. Bolouki, and M. Siami, "More details on analysis of fractional-order Van der Pol oscillator," Journal of Vibration and Control, vol. 15, no. 6, pp. 803-819, 2009.

[12] I. Grigorenko and E. Grigorenko, "Chaotic dynamics of the fractional Lorenz system," Physical Review Letters, vol. 91, no. 3, Article ID 034101, 2003.

[13] Y. Yu, H.-X. Li, S. Wang, and J. Yu, "Dynamic analysis of a fractional-order Lorenz chaotic system," Chaos, Solitons and Fractals, vol. 42, no. 2, pp. 1181-1189, 2009.

[14] C. Li and G. Chen, "Chaos in the fractional order Chen system and its control," Chaos, Solitons and Fractals, vol. 22, no. 3, pp. 549-554, 2004.

[15] J. G. Lu and G. Chen, "A note on the fractional-order Chen system," Chaos, Solitons and Fractals, vol. 27, no. 3, pp. 685-688, 2006.

[16] T. Ueta and G. Chen, "Bifurcation analysis of Chen's equation," International Journal of Bifurcation and Chaos in Applied Sciences and Engineering, vol. 10, no. 8, pp. 1917-1931, 2000.

[17] J. G. Lu, "Chaotic dynamics of the fractional-order Lü system and its synchronization," Physics Letters A, vol. 354, no. 4, pp. 305-311, 2006. 
[18] X.-Y. Wang and M.-J. Wang, "Dynamic analysis of the fractional-order Liu system and its synchronization," Chaos, vol. 17, no. 3, Article ID 033106, 2007.

[19] C. Li and G. Chen, "Chaos and hyperchaos in the fractionalorder Rössler equations," Physica A, vol. 341, no. 1-4, pp. 55-61, 2004.

[20] W. Zhang, S. Zhou, H. Li, and H. Zhu, "Chaos in a fractionalorder Rössler system," Chaos, Solitons and Fractals, vol. 42, no. 3, pp. 1684-1691, 2009.

[21] J. G. Lu, "Chaotic dynamics and synchronization of fractionalorder Arneodo's systems," Chaos, Solitons and Fractals, vol. 26, no. 4, pp. 1125-1133, 2005.

[22] E. Ahmed, A. M. A. El-Sayed, and H. A. A. El-Saka, "Equilibrium points, stability and numerical solutions of fractionalorder predator-prey and rabies models," Journal of Mathematical Analysis and Applications, vol. 325, no. 1, pp. 542-553, 2007.

[23] I. Petras, Fractional-Order Nonlinear Systems, Springer, Berlin, Germany, 2011.

[24] W.-C. Chen, "Nonlinear dynamics and chaos in a fractionalorder financial system," Chaos, Solitons and Fractals, vol. 36, no. 5, pp. 1305-1314, 2008.

[25] M. S. Tavazoei and M. Haeri, "Chaotic attractors in incommensurate fractional order systems," Physica D, vol. 237, no. 20, pp. 2628-2637, 2008.

[26] G. C. Wu and D. Baleanu, "Discrete fractional logistic map and its chaos," Nonlinear Dynamics, 2013.

[27] C. P. Li, W. H. Deng, and D. Xu, "Chaos synchronization of the Chua system with a fractional order," Physica A, vol. 360, no. 2, pp. 171-185, 2006.

[28] C. Li and J. Yan, "The synchronization of three fractional differential systems," Chaos, Solitons \& Fractals, vol. 32, no. 2, pp. 751-757, 2007.

[29] Z. M. Odibat, N. Corson, M. A. Aziz-Alaoui, and C. Bertelle, "Synchronization of chaotic fractional-order systems via linear control," International Journal of Bifurcation and Chaos in Applied Sciences and Engineering, vol. 20, no. 1, pp. 81-97, 2010.

[30] S. Kuntanapreeda, "Robust synchronization of fractional-order unified chaotic systems via linear control," Computers \& Mathematics with Applications, vol. 63, no. 1, pp. 183-190, 2012.

[31] G. Peng, "Synchronization of fractional order chaotic systems," Physics Letters A, vol. 363, no. 5-6, pp. 426-432, 2007.

[32] L.-G. Yuan and Q.-G. Yang, "Parameter identification and synchronization of fractional-order chaotic systems," Communications in Nonlinear Science and Numerical Simulation, vol. 17, no. 1, pp. 305-316, 2012.

[33] A. S. Hegazi, E. Ahmed, and A. E. Matouk, "On chaos control and synchronization of the commensurate fractional order Liu system," Communications in Nonlinear Science and Numerical Simulation, vol. 18, no. 5, pp. 1193-1202, 2013.

[34] H. Zhu, S. Zhou, and Z. He, "Chaos synchronization of the fractional-order Chen's system," Chaos, Solitons and Fractals, vol. 41, no. 5, pp. 2733-2740, 2009.

[35] A. E. Matouk, "Chaos, feedback control and synchronization of a fractional-order modified autonomous Van der Pol-Duffing circuit," Communications in Nonlinear Science and Numerical Simulation, vol. 16, no. 2, pp. 975-986, 2011.

[36] J. Yan and C. Li, "On chaos synchronization of fractional differential equations," Chaos, Solitons \& Fractals, vol. 32, no. 2, pp. 725-735, 2007.
[37] S. Bhalekar and V. Daftardar-Gejji, "Synchronization of different fractional order chaotic systems using active control," Communications in Nonlinear Science and Numerical Simulation, vol. 15, no. 11, pp. 3536-3546, 2010.

[38] M. M. Asheghan, M. T. H. Beheshti, and M. S. Tavazoei, "Synchronization and anti-synchronization of new uncertain fractional-order modified unified chaotic systems via novel active pinning control," Communications in Nonlinear Science and Numerical Simulation, vol. 16, no. 2, pp. 1044-1051, 2011.

[39] L. Pan, W. Zhou, L. Zhou, and K. Sun, "Chaos synchronization between two different fractional-order hyperchaotic systems," Communications in Nonlinear Science and Numerical Simulation, vol. 16, no. 6, pp. 2628-2640, 2011.

[40] Z. Odibat, "A note on phase synchronization in coupled chaotic fractional order systems," Nonlinear Analysis: Real World Applications, vol. 13, no. 2, pp. 779-789, 2012.

[41] M. Mahmoudian, R. Ghaderi, A. Ranjbar, J. Sadati, S. H. Hosseinnia, and S. Momani, "Synchronization of fractionalorder chaotic system via adaptive PID controller," in New Trends in Nanotechnology and Fractional Calculus Applications, pp. 445-452, Springer, 2010.

[42] L. Jie, L. Xinjie, and Z. Junchan, "Prediction-control based feedback control of a fractional order unified chaotic system," in Proceedings of the Chinese Control and Decision Conference (CCDC '11), pp. 2093-2097, May 2011.

[43] Y. B. Zhang, W. G. Sun, J. W. Wang, and K. Yang, "Mixed synchronization for a class of fractionalorder chaotic systems via open-plus-closed-loop control," Communications in Fractional Calculus, vol. 3, pp. 62-72, 2012.

[44] C. Yin, S.-m. Zhong, and W.-f. Chen, "Design of sliding mode controller for a class of fractional-order chaotic systems," Communications in Nonlinear Science and Numerical Simulation, vol. 17, no. 1, pp. 356-366, 2012.

[45] M. P. Aghababa, "Robust stabilization and synchronization of a class of fractional-order chaotic systems via a novel fractional sliding mode controller," Communications in Nonlinear Science and Numerical Simulation, vol. 17, no. 6, pp. 2670-2681, 2012.

[46] S. Dadras and H. R. Momeni, "Control of a fractional-order economical system via sliding mode," Physica A, vol. 389, no. 12, pp. 2434-2442, 2010.

[47] J. Yuan, S. Bao, and J. Wenqiang, "Adaptive sliding mode control of a novel class of fractional chaotic systems," Advances in Mathematical Physics, vol. 2013, Article ID 576709, 13 pages, 2013.

[48] M. R. Faieghi, H. Delavari, and D. Baleanu, "Control of an uncertain fractional-order Liu system via fuzzy fractional-order sliding mode control," Journal of Vibration and Control, vol. 18, no. 9, pp. 1366-1374, 2012.

[49] M. R. Faieghi, H. Delavari, and D. Baleanu, "A note on stability of sliding mode dynamics in suppression of fractional-order chaotic systems," Computers \& Mathematics with Applications, vol. 66, no. 5, pp. 832-837, 2013.

[50] J. Yuan, S. Bao, and D. Chao, "Pseudo-state sliding mode control of fractional SISO nonlinear systems," Advances in Mathematical Physics. In press.

[51] C. Yin, S. Dadras, S.-M. Zhong, and Y. Chen, "Control of a novel class of fractional-order chaotic systems via adaptive sliding mode control approach," Applied Mathematical Modelling, vol. 37, no. 4, pp. 2469-2483, 2013.

[52] R. Caponetto, Fractional Order Systems: Modeling and Control Applications, vol. 72, World Scientific Publishing Company, 2010. 
[53] C. A. Monje, Y. Chen, B. M. Vinagre, D. Xue, and V. Feliu, Fractional-Order Systems and Controls: Fundamentals and Applications, Springer, London, UK, 2010.

[54] J. Lü, G. Chen, D. Cheng, and S. Celikovsky, "Bridge the gap between the Lorenz system and the Chen system," International Journal of Bifurcation and Chaos in Applied Sciences and Engineering, vol. 12, no. 12, pp. 2917-2926, 2002.

[55] D. Baleanu, K. Diethelm, E. Scalas, and J. J. Trujillo, Fractional Calculus: Models and Numerical Methods, Series on Complexity, Nonlinearity and Chaos, World Scientific, Singapore, 2012.

[56] X. Wu, J. Li, and G. Chen, "Chaos in the fractional order unified system and its synchronization," Journal of the Franklin Institute, vol. 345, no. 4, pp. 392-401, 2008. 


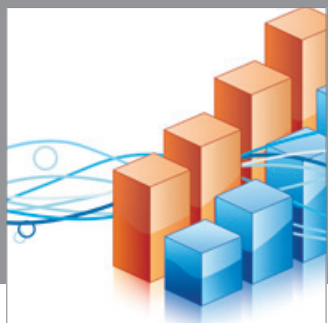

Advances in

Operations Research

mansans

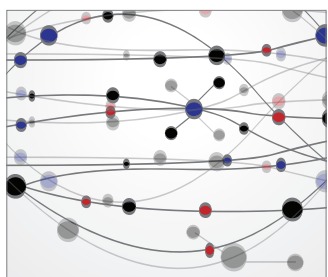

The Scientific World Journal
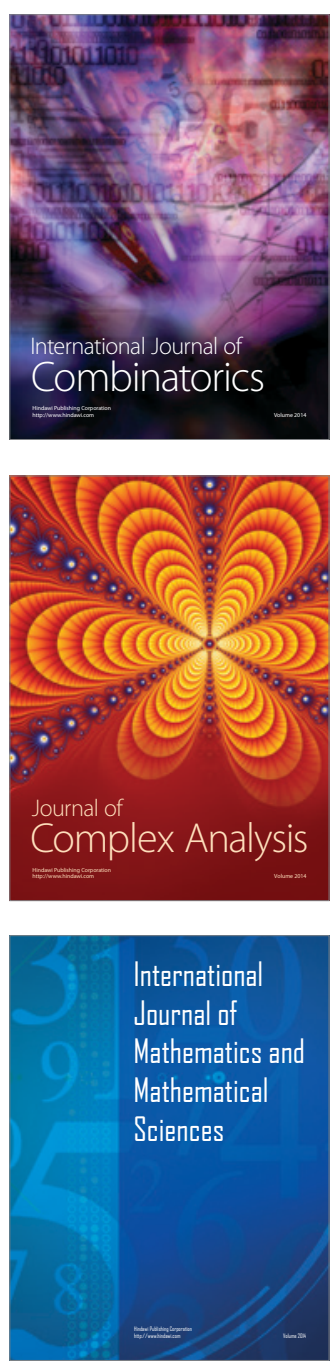
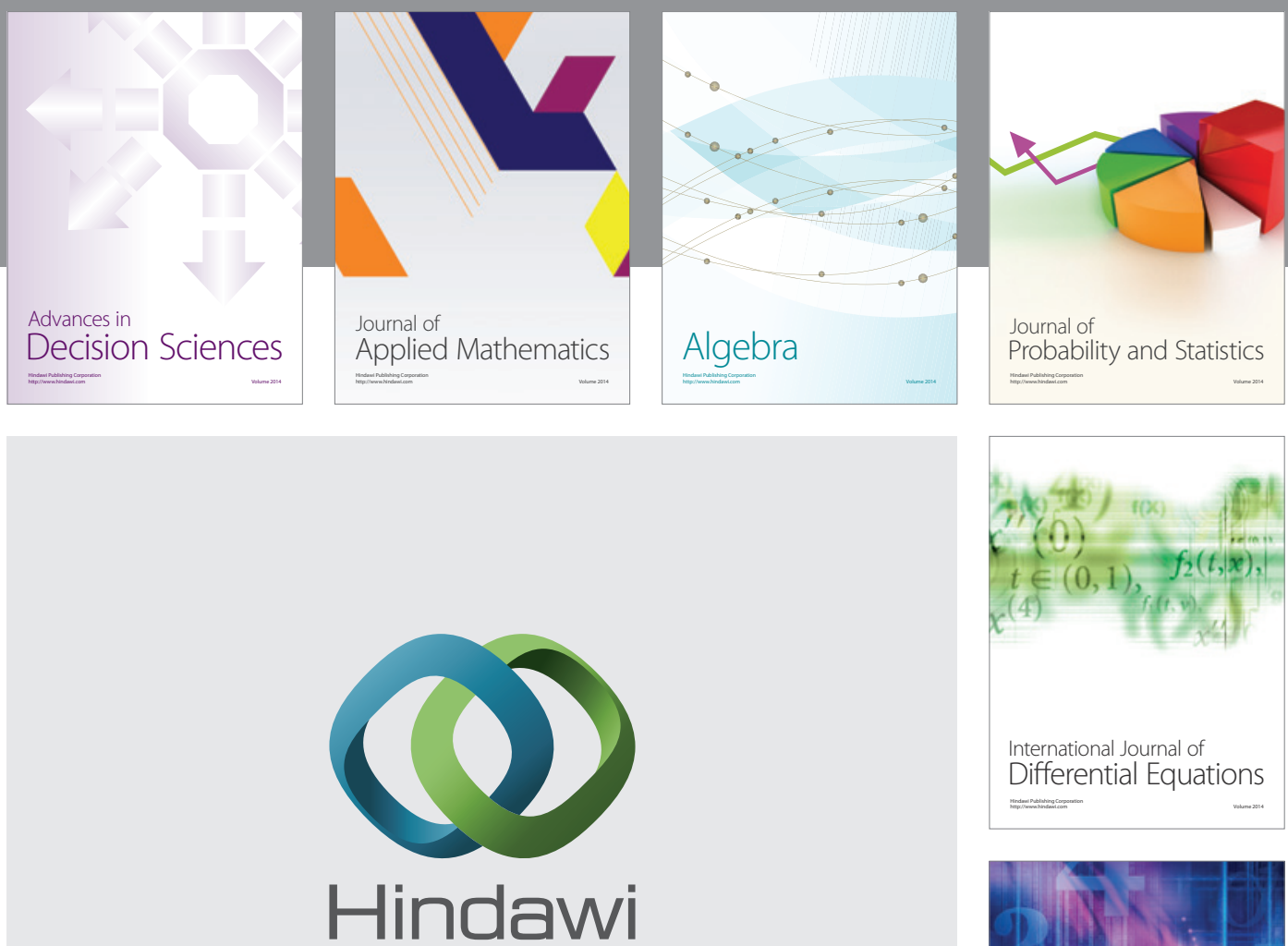

Submit your manuscripts at http://www.hindawi.com
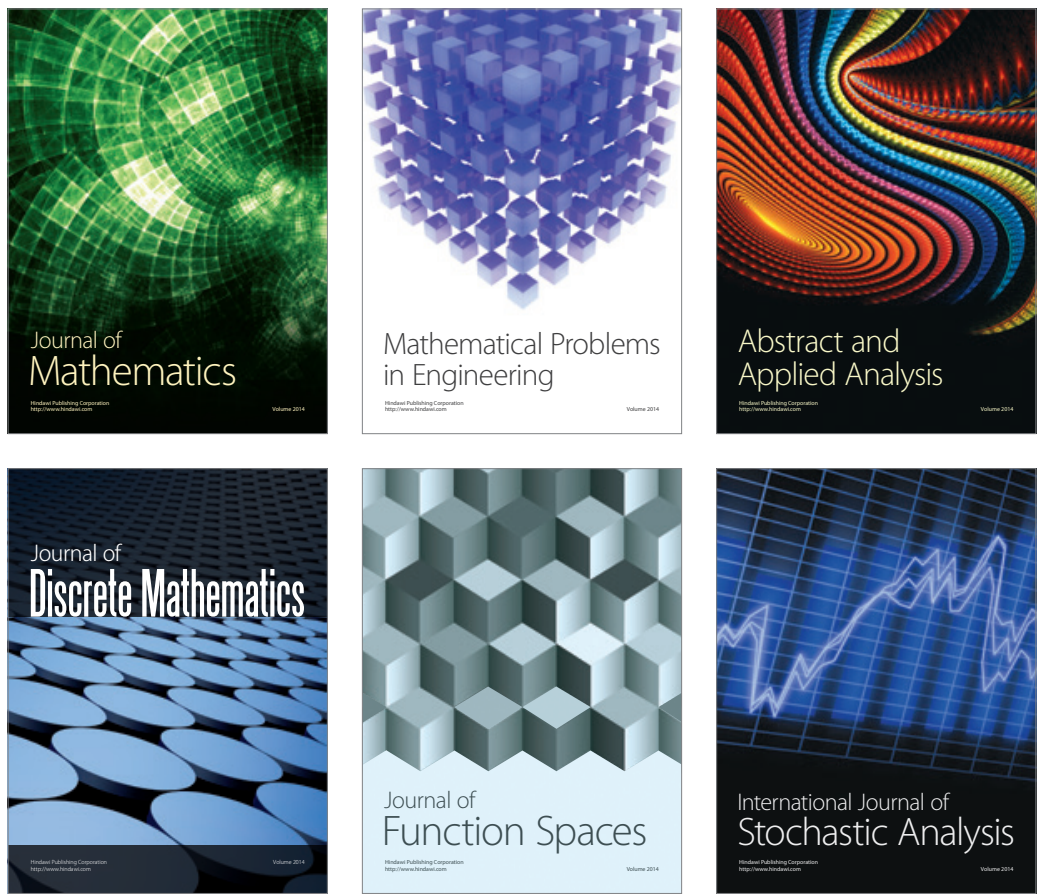

Journal of

Function Spaces

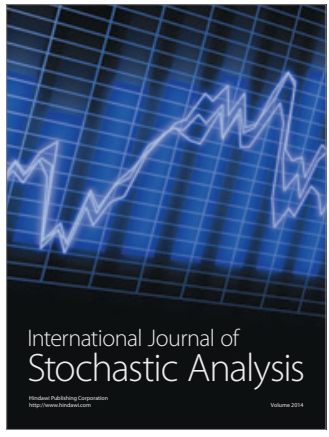

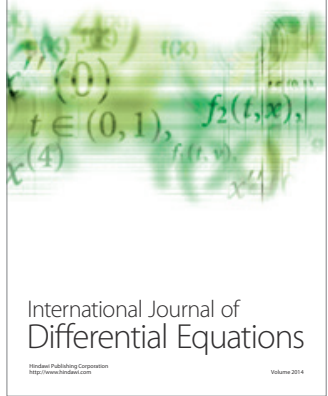
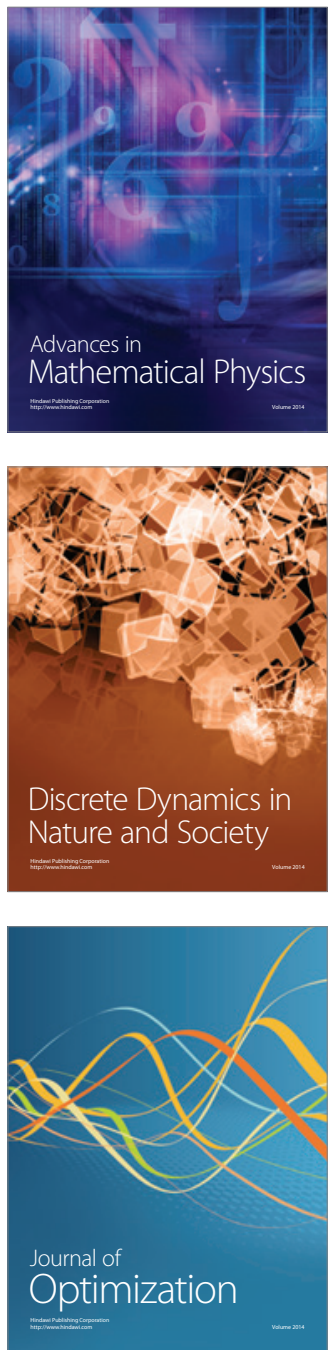